\title{
Characteristics of the double-cycled motion-ruled surface of the Schatz linkage based on differential geometry
}

\author{
Lei Cui', Jian S Dai ${ }^{2}$ and Chung-Ching Lee ${ }^{3}$
}

\begin{abstract}
This paper applies Euclidean invariants from differential geometry to kinematic properties of the ruled surfaces generated by the coupler link and the constraint-screw axes. Starting from investigating the assembly configuration, the work reveals two cycle phases of the coupler link when the input link finishes a full rotation. This leads to analysis of the motion ruled surface generated by the directrix along the coupler link, where Euclidean invariants are obtained and singularities are identified. This work further presents the constraint ruled surface that is generated by the constraint screw axes and unveils its intrinsic characteristics.
\end{abstract}

\section{Keywords \\ Over-constrained mechanism, ruled surface, Euclidean invariants, differential geometry}

Date received: II October 20I3; accepted: 16 May 2014

\section{Introduction}

Schatz linkage was discovered as Paul Schatz unveiled in 1929 the invertible cube from the Platonic solids. The invertible cube has the astounding property of invertibility while the Schatz mechanism provides a perfect dynamic balance of both centripetal and centrifugal forces as Turbula patented in 1971 as the only widely-used overconstrained linkages in industry, mainly used as spatial mixing-machines. ${ }^{1}$

The Schatz linkage consists of six revolute joints and is able to move in a three-dimensional space. As one of the overconstrained mechanisms which have mobility over a finite range of motion without satisfying the Grübler-Kutzbach mobility criterion, the motion is generated from a special geometric arrangement of joint axes. Study of overconstrained mechanisms and their geometric properties has inspired many engineers and researchers over the past one and half centuries. In 1853, Sarrus $^{2}$ presented the first spatial overconstrained linkage with two sets of joints each of which consists of three parallel joints. In 1903, Bennett $^{3}$ put forward a spatial $4 \mathrm{R}$ linkage with each joint axis perpendicular to the two adjacent links connected and in 1905 he proposed the condition ${ }^{4}$ for the linkage to have a single degree of mobility. This led to a $6 \mathrm{R}$ overconstrained linkage ${ }^{5}$ by combining two spherical 4R linkages. In 1927, Bricard ${ }^{6}$ identified six mobile 6R overconstrained linkages including the line-symmetric loop, plane-symmetric loop, orthogonal chain, plane-symmetric octahedral chain, doubly-collapsible octahedral chain, and plano-spherical hybrid chain. In 1931, Myard ${ }^{7}$ produced a planesymmetric overconstrained 5R linkage. In 1943, Goldberg ${ }^{8}$ obtained a 5R overconstrained linkage by combining a pair of Bennett linkages in a way that a link common to both was removed and a pair of adjacent links were rigidly attached to each other. In 1954, Altmann ${ }^{9}$ presented a $6 \mathrm{R}$ linkage which was a special case of the Bricard line-symmetric linkage. In 1968, Waldron $^{10}$ suggested that any two single-loop linkages with a single degree of freedom could be arranged in space to make them to share a common axis and further exemplified a $6 \mathrm{R}$ overconstrained mechanism made from two Bennett linkages. The mechanism is connected in a way that each of the Bennett linkages has a revolute joint that is collinear to the other. In 1975, Schatz ${ }^{11}$ patented Schatz linkage which was identified as a special trihedral Bricard linkage.

\footnotetext{
'Department of Mechanical Engineering, Curtin University, Perth, Australia

${ }^{2}$ Centre for Robotics Research, King's College London, University of London, London, UK

${ }^{3}$ Department of Mechanical Engineering, National Kaohsiung University of Applied Sciences, Kaohsiung, Taiwan
}

Corresponding author:

Jian S Dai, Kings College London, King's College London Strand London, WC2R 2LS United Kingdom.

Email: Jian.Dai@KCL.ac.uk 
As a typical type of 6R overconstrained mechanisms which were investigated by Mavroidis and Roth, ${ }^{12}$ Baker, ${ }^{13}$ Cui and Dai, ${ }^{14}$ the kinematics and dynamics of the Schatz linkage attracted attention of several researchers for quite sometime. ${ }^{15-19}$ A closedform solution and synthesis of this mechanism were established by matrix and optimization approach. Further using the Denavit-Hartenberg approach, Lee $^{17}$ and Lee and Dai ${ }^{18}$ presented algebraic equations of the motion. ${ }^{20}$

The insight of the coupler motion surface, particularly its innate cycle phases, has not yet been revealed in this study. The most startling feature of the Schatz linkage rests on its coupler which generates a random motion with its vibrant character that makes this linkage attractive and makes the first use of an overconstrained mechanism ${ }^{12-14}$ in the industry. The coupler link of the Schatz linkage produces a combination of rotating, tumbling, and shaking movement, a unique feature that has been utilized in mixer or blender when the coupler link takes a spatial trajectory. The closedloop linkage effectively transforms an input from the crank into an "inversion" motion in an elegant way.

The coupler takes a full revolution but the axis in line with this coupler takes two revolution with two cycles and generates a double-butterfly ruled surface. To reveal the motion particularly the motion-ruled surface of the coupler, this paper takes differential geometry together with screw system analysis on overconstrained mechanisms ${ }^{21}$ and reveals the different phase cycles of the coupler when it rolls a full revolution. While Euclidean invariants ${ }^{22-28}$ remain identical with respect to coordinate transformations, the ruled surface of the coupler can be investigated at this point of view. This leads to the intrinsic geometric features of the motion and the constraint ruled surface.

As such, the ruled surface generated by the coupler link directly reflects the intrinsic motion properties of the Schatz linkage. On the other hand, the constraint screw of the linkage exhibits the geometric features of the force applied to the coupler link.

\section{Assembly configurations}

For a general spatial close-loop linkage consisting of all revolute joints having one degree of freedom, seven joints are the minimum number required. With respect to the Schatz linkage that has only six revolution joints, certain geometric constraints are to be satisfied for being mobile. This leads to the dimension constraints with two assembly configurations.

The Schatz linkage in Figure 1 requires that the links $A_{2} A_{3}, B_{2} B_{3}$, and $A_{3} B_{3}$ have the same length $a$ and the length of link $A_{1} B_{1}$ is $\sqrt{3} a$. The links $A_{1} A_{2}$ and $B_{1} B_{2}$ have the same length $d$. Further, each of the joint axes from $A_{2}$ to $B_{2}$ is perpendicular to its adjacent two axes and joint axis $A_{1}$ is parallel to $B_{1}$. Link $A_{3} B_{3}$ is termed coupler link in the paper.
The coupler link $A_{3} B_{3}$ in Figure 1 is constrained by two limbs: $A_{1} A_{2} A_{3}$ and $B_{1} B_{2} B_{3}$. If these two limbs are considered as open-chain mechanisms, the position vectors $O A_{3}$ that is represented by $\boldsymbol{r}_{1}$ and $O B_{3}$ represented by $\boldsymbol{r}_{2}$, and the direction vectors $\boldsymbol{z}_{A 3}$ and $\boldsymbol{z}_{B 3}$ can be obtained. The key observation is that $\boldsymbol{r}_{1}$ and $\boldsymbol{z}_{A 3}$ are functions of the joint angles $\theta_{1}$ and $\theta_{2} ; \boldsymbol{r}_{2}$ and $\boldsymbol{z}_{B 3}$ are functions of the joint angles $\theta_{5}$ and $\theta_{6}$-the joint angles $\theta_{3}$ and $\theta_{4}$ are not involved. The geometry of the Schatz linkage gives four constraints

$$
\left\{\begin{array}{l}
\left\|\boldsymbol{r}_{1}-\boldsymbol{r}_{2}\right\|^{2}=a^{2} \\
\boldsymbol{z}_{A 3} \cdot \boldsymbol{z}_{B 3}=0 \\
\boldsymbol{z}_{B 3} \cdot\left(\boldsymbol{r}_{1}-\boldsymbol{r}_{2}\right)=0 \\
\boldsymbol{z}_{A 3} \cdot\left(\boldsymbol{r}_{1}-\boldsymbol{r}_{2}\right)=0
\end{array}\right.
$$

These four constraints yield four nonlinear equations of the four joint angles: $\theta_{1}, \theta_{2}, \theta_{5}$, and $\theta_{6}$

$$
\begin{aligned}
& \sqrt{3}\left(\cos \theta_{1} \cos \theta_{2}-\cos \theta_{6} \cos \theta_{5}\right) \\
& \quad-\cos \theta_{2} \cos \theta_{5} \cos \left(\theta_{1}-\theta_{6}\right) \\
& \quad-\sin \theta_{2} \sin \theta_{5}+2=0 \\
& \sin \theta_{2} \sin \theta_{5} \cos \left(\theta_{1}-\theta_{6}\right)+\cos \theta_{2} \cos \theta_{5}=0 \\
& -\sin \theta_{2} \cos \theta_{5} \cos \left(\theta_{1}-\theta_{6}\right)+\sqrt{3} \cos \theta_{1} \sin \theta_{2} \\
& \quad+\cos \theta_{2} \sin \theta_{5}=0 \\
& \cos \theta_{2} \sin \theta_{5} \cos \left(\theta_{1}-\theta_{6}\right)+\sqrt{3} \cos \theta_{6} \sin \theta_{5} \\
& \quad-\sin \theta_{2} \cos \theta_{5}=0
\end{aligned}
$$

This set of nonlinear equations is simplified by the relation between the joint angles $\theta_{2}$ and $\theta_{5}$

$$
\sin \theta_{2} \sin \theta_{5}=\frac{1}{2}
$$

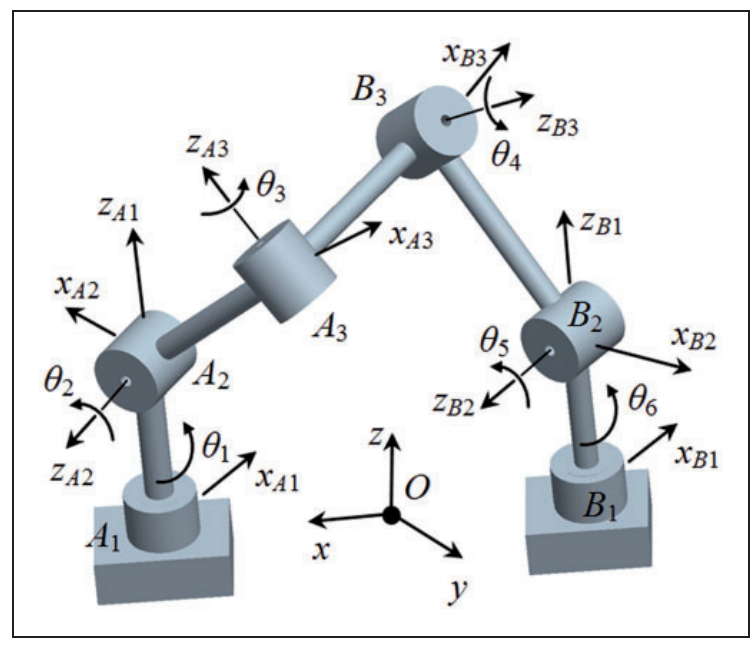

Figure I. Coordinate frames of the Schatz linkage. 
Subsequently, the closed-form solutions of $\theta_{2}, \theta_{5}$, and $\theta_{6}$ in terms of $\theta_{1}$ are obtained. A further investigation reveals two assembly configurations as follows. Assembly configuration 1 is produced when the following is satisfied as

$$
\begin{aligned}
& \left\{\begin{array}{l}
\sin \theta_{2}=\frac{1}{2} \sqrt{4-3 \cos ^{2} \theta_{1}} \\
\cos \theta_{2}=-\frac{\sqrt{3}}{2} \cos \theta_{1}
\end{array},\left\{\begin{array}{l}
\sin \theta_{5}=\frac{1}{\sqrt{4-3 \cos ^{2} \theta_{1}}} \\
\cos \theta_{5}=\frac{\sqrt{3} \sin \theta_{1}}{\sqrt{4-3 \cos ^{2} \theta_{1}}}
\end{array}\right.\right. \\
& \left\{\begin{array}{l}
\sin \theta_{6}=\frac{\cos \theta_{1}}{\sqrt{4-3 \cos ^{2} \theta_{1}}} \\
\cos \theta_{6}=\frac{2 \sin \theta_{1}}{\sqrt{4-3 \cos ^{2} \theta_{1}}}
\end{array}\right.
\end{aligned}
$$

Assembly configuration 2 is produced when the following is satisfied as

$$
\begin{aligned}
& \left\{\begin{array}{l}
\sin \theta_{2}=-\frac{1}{2} \sqrt{4-3 \cos ^{2} \theta_{1}} \\
\cos \theta_{2}=-\frac{\sqrt{3}}{2} \cos \theta_{1}
\end{array},\left\{\begin{array}{l}
\sin \theta_{5}=-\frac{1}{\sqrt{4-3 \cos ^{2} \theta_{1}}} \\
\cos \theta_{5}=\frac{\sqrt{3} \sin \theta_{1}}{\sqrt{4-3 \cos ^{2} \theta_{1}}}
\end{array}\right.\right. \\
& \left\{\begin{array}{l}
\sin \theta_{6}=\frac{\cos \theta_{1}}{\sqrt{4-3 \cos ^{2} \theta_{1}}} \\
\cos \theta_{6}=\frac{2 \sin \theta_{1}}{\sqrt{4-3 \cos ^{2} \theta_{1}}}
\end{array}\right.
\end{aligned}
$$

It can be seen that the link length $d$ of link $A_{1}$ and $A_{2}$ and link $B_{1}$ and $B_{2}$ has no influence on motion of the other three links, so it can be set to 0 to reduce the inertial effect. In this case, joints $A_{1}$ and $A_{2}$ merge into a universal joint, so do joints $B_{1}$ and $B_{2}$. The commercial products "Turbula" have adopted this design.

The values of $\theta_{2}, \theta_{5}$, and $\theta_{6}$ with the values of $\theta_{1}$ varying from 0 to $2 \pi$ in these two assembly configurations are shown in Figure 2.

From Figure 2, it can be seen that the value of the joint variable $\theta_{6}$ remains unchanged and link $B_{1} B_{2}$ also has full rotability. The values of the joint variables $\theta_{2}$ and $\theta_{5}$ change to $-\theta_{2}$ and $-\theta_{5}$.

The solutions in Figure 2 give two assembly configurations. The linkage in assembly configuration 1 is shown in Figure 1 and that in assembly configuration 2 is shown in Figure 3. Note that Schatz linkage cannot change from one configuration to another without being disassembled and reassembled, resulting in two assembly configurations.

The two assembly configurations are produced in the form of elbow up and down but need to be disassembled and reassembled when they change from one to another. Investigating one assembly configuration, it would have the mirror reflection in another assembly configuration.

\section{Cycle phases}

When the input angle $\theta_{1}$ varies in the range of 0 to $2 \pi$, the coupler link experiences two cycle phases. If the coupler link is considered as a line, the two cycle phases are exactly the same. On the other hand, if the coupler link is considered as a rigid body, i.e. with a spatial reference frame attached to it, the two cycle phases become different.

The linkage consists of two limbs $A_{1} A_{2} A_{3}$ and $B_{1} B_{2} B_{3}$ connected by the coupler $A_{3} B_{3}$ link. The end points $A_{3}$ of the first limb and $B_{3}$ of the second limb have two degrees of freedom and generates two spheres. The length of $A_{3} B_{3}$ link restricts the two end points to two closed curves. Thus given one length, two sets of $A_{3}$ and $B_{3}$ can be found in Figure 4.

The closed curve is completed by the input angle $\theta_{1}$ from 0 to $\pi$ and a point in one curve is related to a point in the opposite closed curve by link length of the coupler link. The movement of both endpoints $A_{3}$ and $B_{3}$ of the coupler link completes one phase when the curve is closed while input angle $\theta_{1}$ ranges from 0 to $\pi$. This indicatrix $e_{1}$ as a unit vector

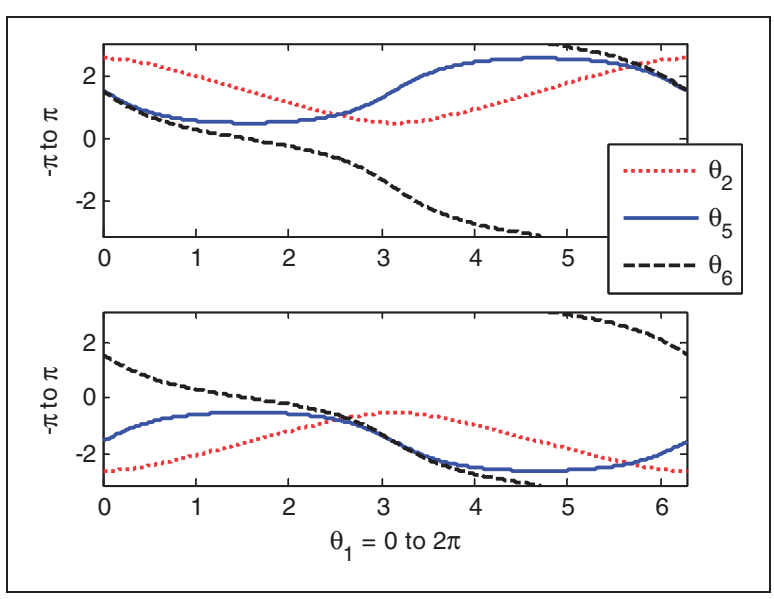

Figure 2. Values of $\theta_{2}, \theta_{5}$, and $\theta_{6}$ with respect to input $\theta_{1}$.

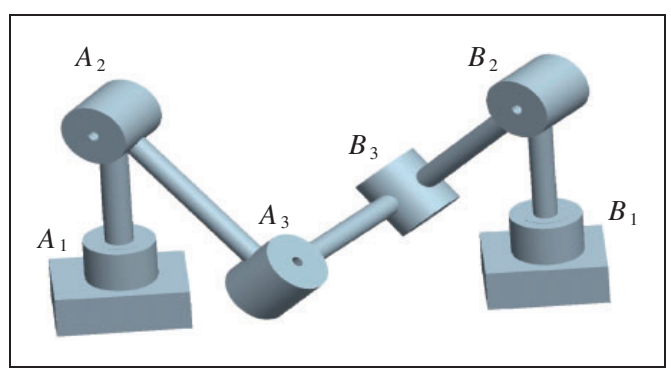

Figure 3. The Schatz linkage in configuration 2. 
along the coupler link $A_{3}$ and $B_{3}$ can be given as follows

$$
\boldsymbol{e}_{1}=\left[\begin{array}{c}
-\frac{\sqrt{3}}{2} \frac{1+3 \sin ^{4} \theta_{1}}{4-3 \cos ^{2} \theta_{1}} \\
\frac{\sqrt{3}}{2} \cos \theta_{1} \sin \theta_{1} \frac{3+3 \sin ^{2} \theta_{1}}{4-3 \cos ^{2} \theta_{1}} \\
\frac{1-3 \sin ^{2} \theta_{1}}{2 \sqrt{4-3 \cos ^{2} \theta_{1}}}
\end{array}\right]
$$

When the input angle $\theta_{1}$ reaches $\pi$, the indicatrix $\boldsymbol{e}_{1}$ along the coupler link $A_{3}$ and $B_{3}$ returns to its starting position and orientation. This completes the first cycle phase.

The second phase starts with the same closed curve when input angle $\theta_{1}$ is at $\pi$. It ends when the input angle reaches $2 \pi$ while the coupler link as a rigid body has a full rotation.

These two phases are illustrated in Figure 5. The rectangular prism on the right serves as the crank, on

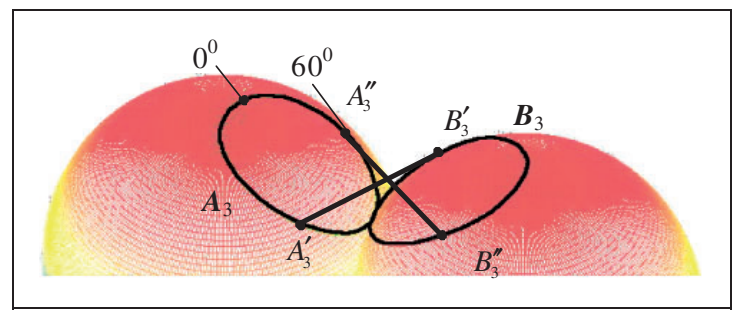

Figure 4. Geometric interpretation of two phases. which " 0 " is marked as seen in Figure 5(a). A frame $x_{c} z_{\mathrm{c}}$ is attached to the coupler link. When the crank rotates a half turn, i.e. $0^{\circ}$ to $180^{\circ}$, the frame $x_{c} z_{\mathrm{c}}$ changes to the position shown in Figure 5(b). This means the coupler link does not return to its initial configuration. Another half-turn has to be provided from the crank for it to return to its initial configuration. These two phases of the coupler link have never been revealed before.

\section{Motion ruled surface of the coupler link}

The indicatrix $\boldsymbol{e}_{1}$ along the coupler link $A_{3} B_{3}$ sweeps out a ruled surface as shown in Figure 6. The parametric form of this ruled surface can be defined as

$$
\boldsymbol{S}\left(\theta_{1}, t\right)=\boldsymbol{r}_{a}\left(\theta_{1}\right)+t \boldsymbol{e}_{1}\left(\theta_{1}\right)
$$

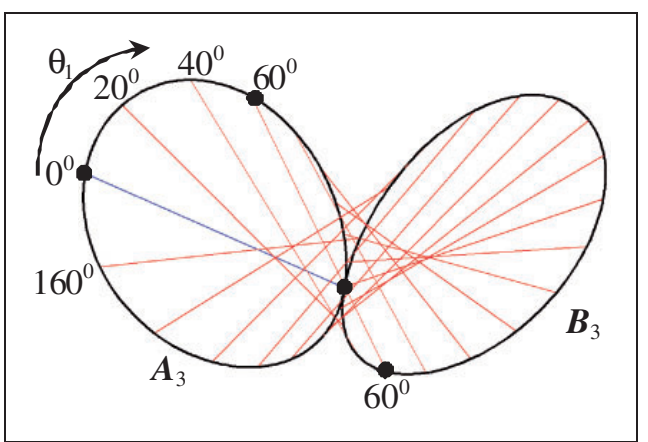

Figure 6. The motion ruled surface of the coupler link.

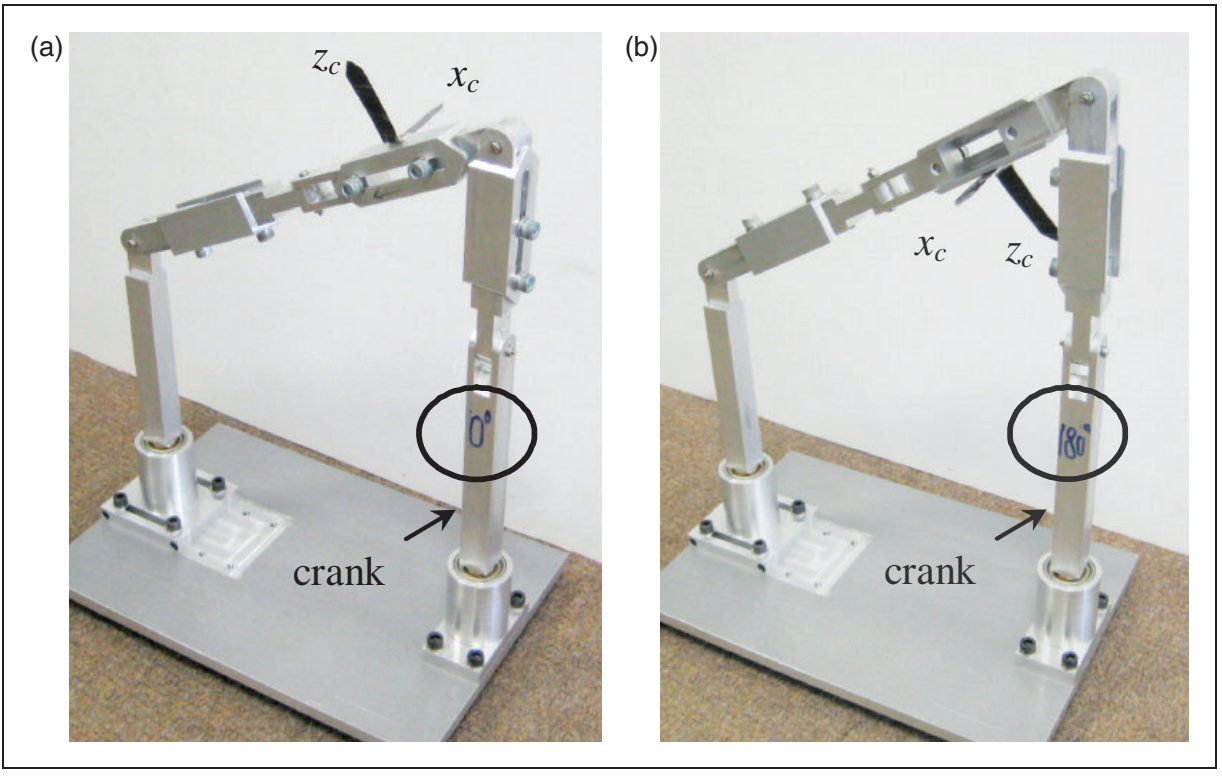

Figure 5. (a) The configuration of the coupler as a rigid body when $\theta_{1}$ is at 0 ; (b) configuration of the coupler as a rigid body when $\theta_{1}$ is at $\pi$. 
where the ruled surface directrix $\boldsymbol{r}_{a}$ gives the base curve at point $A_{3}$ and is given as

$$
\boldsymbol{r}_{a}=\left[\begin{array}{c}
\frac{\sqrt{3}}{2} a \sin ^{2} \theta_{1} \\
-\frac{\sqrt{3}}{2} a \sin \theta_{1} \cos \theta_{1} \\
\frac{a}{2} \sqrt{4-3 \cos ^{2} \theta_{1}}+d
\end{array}\right]
$$

The motion ruled surface traced by the directrix $\boldsymbol{e}_{1}$ following input angle variation from 0 to $2 \pi$ is presented in Figure 6.

The base curve $\boldsymbol{r}_{a}$ as directrix of the ruled surface traced by point $A_{3}$ is illustrated as the closed curve in the left. When the input variable $\theta_{1}$ is at $0^{0}, A_{3}$ is the point at the far left in Figure 6.

The straight-line indicatrix $\boldsymbol{e}_{1}$ of the ruled surface in equation (14) can also be mapped by Gauss map to a corresponding point on a unit sphere. The spherical image of the indicatrix $e_{1}$ is shown in Figure 7.

Geodesic curvature $\beta$ of the spherical indicatrix of $\boldsymbol{e}_{1}$ is the intrinsic parameter. ${ }^{29}$ Its values are shown in Figure 8 in terms of the input variable $\theta_{1}$.

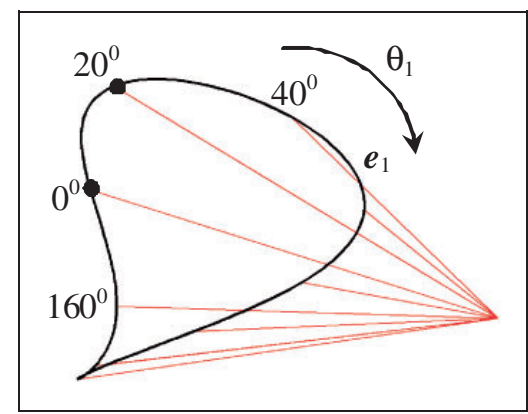

Figure 7. Gauss map of the indicatrix $\mathbf{e}_{\mathbf{}_{1}}$.

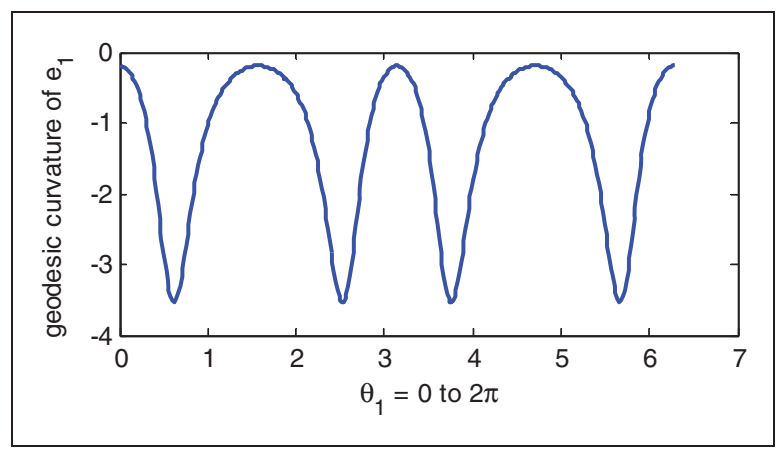

Figure 8. Values of geodesic curvature of the spherical indicatrix $\mathbf{e}_{\text {I }}$ along the coupler link.
A striction curve of the ruled surface consisting of all the central points of a ruled surface can be obtained as

$$
\begin{aligned}
\boldsymbol{M}\left(\theta_{1}\right)= & \boldsymbol{r}_{a}\left(\theta_{1}\right)+u \boldsymbol{e}_{1}\left(\theta_{1}\right) \\
= & {\left[\begin{array}{c}
\frac{\sqrt{3} a}{2} \sin ^{2} \theta_{1} \\
-\frac{\sqrt{3} a}{2} \sin \theta_{1} \cos \theta_{1} \\
\frac{a \sqrt{4-3 \cos ^{2} \theta_{1}}}{2}+d
\end{array}\right] } \\
& +u\left[\begin{array}{c}
-\frac{\sqrt{3}}{2} \frac{1+3 \sin ^{4} \theta_{1}}{4-3 \cos ^{2} \theta_{1}} \\
\frac{\sqrt{3}}{2} \cos \theta_{1} \sin \theta_{1} \frac{3+3 \sin ^{2} \theta_{1}}{4-3 \cos ^{2} \theta_{1}} \\
\frac{1-3 \sin ^{2} \theta_{1}}{2 \sqrt{4-3 \cos ^{2} \theta_{1}}}
\end{array}\right]
\end{aligned}
$$

where $u=-\frac{\dot{\boldsymbol{r}}_{a} \cdot \dot{\boldsymbol{e}}_{1}}{\dot{\boldsymbol{e}}_{1} \cdot \dot{\boldsymbol{e}}_{1}}$. The striction curve $\boldsymbol{M}$ of the ruled surface is given in Figure 9.

It is interesting to note that there are two singular points on the striction curve as in Figure 9. When the input variable $\theta_{1}$ varies from 0 to $\pi / 2$, the striction curve $\boldsymbol{M}$ runs half way as in Figure 9(a). As the input variable $\theta_{1}$ increases from $\pi / 2$ to $\pi$, the striction curve returns to the starting point and completes the first cycle phase. The whole striction curve $\boldsymbol{M}$ is shown in Figure 9(b). Thus, it can be deduced that the striction curve has two singular points when $\theta_{1}$ equals to 0 or $\pi / 2$.

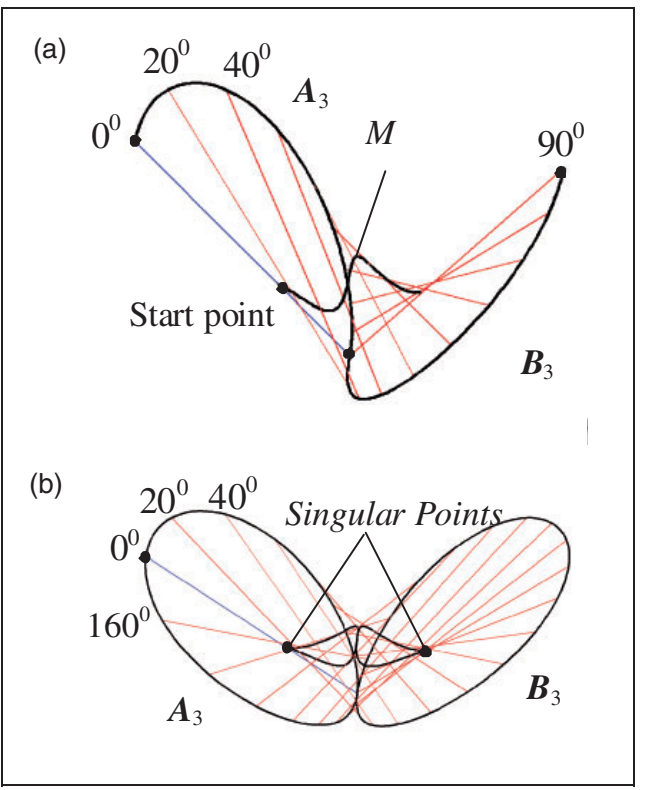

Figure 9. Striction curve of the double-cycled motion-ruled surface: (a) $\theta_{1}$ from 0 to $90^{\circ} ; \theta_{1}$ from 0 to $180^{\circ}$. 


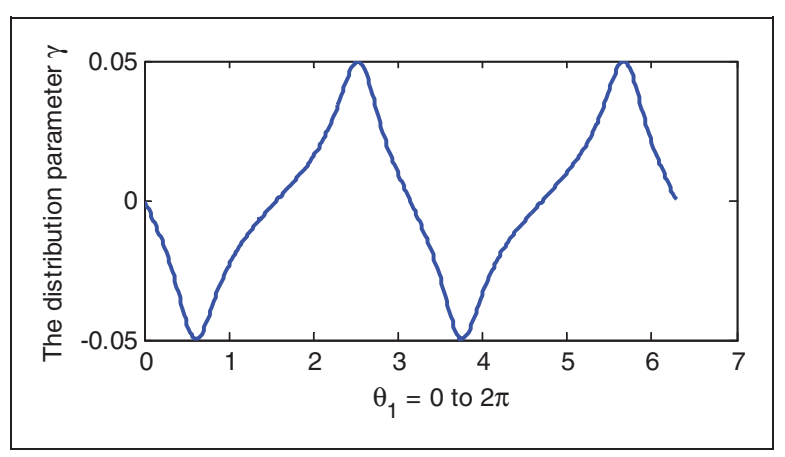

Figure 10. Values of the distribution parameter of the coupler-link rued surface.

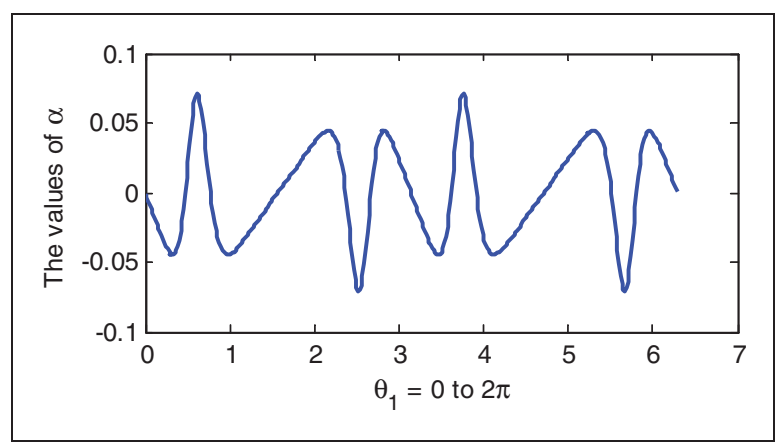

Figure II. Values of $\alpha$ of the coupler-link ruled surface.

It is known from differential geometry that the striction curve could have singular points even if the ruled surface does not necessarily have singularities and it is known that there is no singularity existing for Schatz linkage.

Since the Schatz linkage is symmetrical, the striction curve of the coupler-link ruled surface is also symmetrical, as expected.

Distribution parameter $\gamma$ is the ratio of the distance to the angle of two instantaneously closed generatrix and is

$$
\gamma=\frac{\mathrm{d} \boldsymbol{M}}{\mathrm{d} s} \cdot \boldsymbol{e}_{3}
$$

where $\mathrm{d} s$ is the infinitesimal arc length of the spherical indicatrix, $\boldsymbol{e}_{3}=\mathrm{d}^{2} \boldsymbol{e}_{1} / \mathrm{d} s^{2}$. The values of the distribution parameter $\gamma$ of the coupler-link ruled surface is given in Figure 10.

Similarly, the third Euclidean invariants $\alpha$ can be obtained as $\alpha=\frac{\mathrm{d} \boldsymbol{M}}{\mathrm{d} s} \cdot \boldsymbol{e}_{1}$. The values of $\alpha$ corresponding to the input values of $\theta_{1}$ are shown in Figure 11.

\section{The constraint ruled surface}

Let screws $\$_{1}$ to $\$_{3}$ be associated with joints $A_{1}$ to $A_{3}$, and screws $\$_{6}$ to $\$_{4}$ associated with joints $B_{1}$ to $B_{3}$ in Figure 1. From the closed-form solution equation (7), the direction of each of $\$_{i}$ and a point on $\$_{i}$ can be obtained accordingly. The screw system can thus be given as

$$
\left[\begin{array}{llllll}
\$_{1} & \$_{2} & \$_{3} & \$_{4} & \$_{5} & \$_{6}
\end{array}\right]
$$

where

$$
\$_{1}=\left[\begin{array}{c}
0 \\
0 \\
1 \\
0 \\
-\frac{\sqrt{3} a}{2} \\
0
\end{array}\right], \quad \$_{2}=\left[\begin{array}{c}
s \\
-c \\
0 \\
\mathrm{~d} c \\
-\mathrm{d} s \\
0
\end{array}\right]
$$$$
\$_{3}=\left[\begin{array}{c}
\frac{1}{2} c k \\
\frac{1}{2} s k \\
\frac{\sqrt{3} c}{2} \\
-\frac{1}{2} s(2 a+\mathrm{d} k) \\
\frac{1}{4} c(a+2 \mathrm{~d} k) \\
\frac{\sqrt{3} a}{4} s k
\end{array}\right], \quad \$_{4}=\left[\begin{array}{c}
\frac{2 s}{k^{2}} \\
\frac{c}{k^{2}} \\
-\frac{\sqrt{3} s}{k} \\
\frac{c\left(3 a c^{2}-4 a-\mathrm{d} k\right)}{k^{3}} \\
-\frac{s\left(3 a c^{2}-4 a-4 \mathrm{~d} k\right)}{2 k^{3}} \\
-\frac{\sqrt{3} a c}{2 k^{2}}
\end{array}\right] \text {, }
$$

$$
\$_{5}=\left[\begin{array}{c}
\frac{c}{k} \\
-\frac{2 s}{k} \\
0 \\
\frac{2 \mathrm{~d} s}{k} \\
\frac{\mathrm{d} c}{k} \\
\frac{\sqrt{3} a s}{k}
\end{array}\right], \quad \$_{6}=\left[\begin{array}{c}
0 \\
0 \\
1 \\
0 \\
\frac{\sqrt{3} a}{2} \\
0
\end{array}\right]
$$

where $s, \quad c$, and $k$ represent $\sin \theta_{1}, \cos \theta_{1}$, and $\sqrt{4-3 \cos ^{2} \theta_{1}}$, respectively.

The reciprocal screw can be obtained by the cofactor approach ${ }^{30}$ as follows

$$
\$^{r}=\left[\boldsymbol{w}^{\mathrm{T}}, \boldsymbol{v}^{\mathrm{T}}\right]^{\mathrm{T}}=\left[\begin{array}{c}
-\sqrt{3} k \\
0 \\
\left(3 c^{2}-2\right) \\
-2 \sqrt{3} a s c \\
\frac{\sqrt{3}}{2}\left(a\left(c^{2}-2\right)-2 d k\right) \\
0
\end{array}\right]
$$




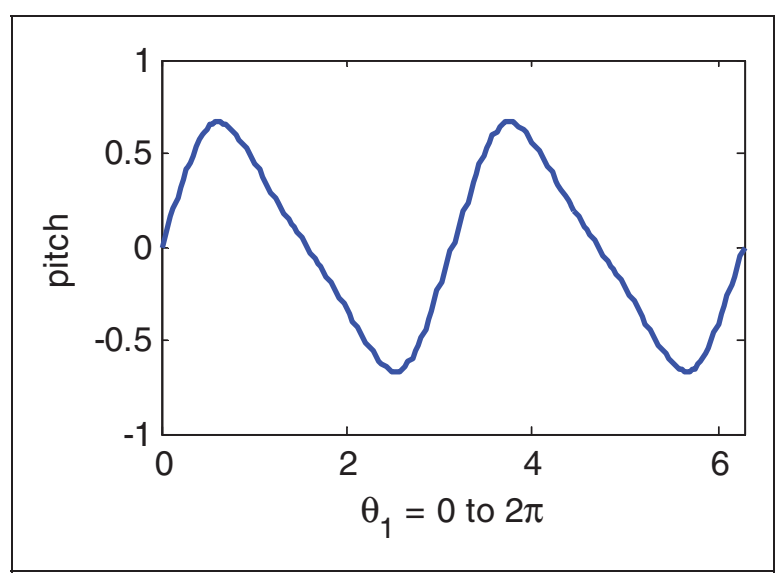

Figure 12. Values of the pitch of the constraint.

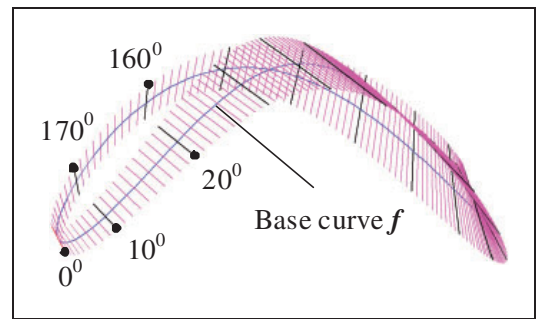

Figure 13. Base curve of the constraint ruled surface.

Its pitch is

$$
p=\frac{\boldsymbol{w} \cdot \boldsymbol{v}}{\boldsymbol{w} \cdot \boldsymbol{w}}=\frac{6 a k s c}{N}
$$

where

$$
N=16-21 c^{2}+9 c^{4}
$$

This pitch is given in Figure 12 when input angle $\theta_{1}$ varies from 0 to $2 \pi$ in a full cycle.

A base curve of the ruled surface can be obtained as

$$
\boldsymbol{f}=\frac{\boldsymbol{w} \times \boldsymbol{v}}{\boldsymbol{w} \cdot \boldsymbol{w}}=\frac{1}{N}\left[\begin{array}{c}
\frac{-2 \sqrt{3}}{3}\left(3 c^{2}-2\right)\left(2 a+2 d k-a c^{2}\right) \\
\frac{8 \sqrt{3}}{3} a\left(3 c^{2}-2\right) s c \\
2\left(k a c^{2}+6 d c^{2}-2 k a-8 d\right)
\end{array}\right]
$$

Thus, the constraint ruled surface with the above base curve is given in Figure 13.

The constraint ruled surface directrix can be obtained as

$$
I_{1}=\frac{w}{\sqrt{w \cdot w}}=\frac{w}{\sqrt{N}}
$$

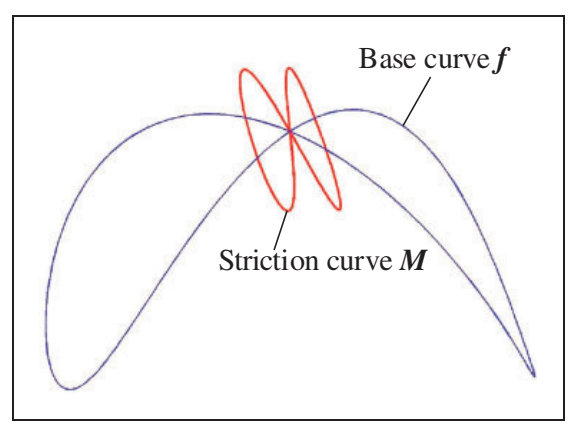

Figure 14. Striction curve of the constraint ruled surface.

The geodesic curvature $\beta$ of $\boldsymbol{I}_{1}$ can further be given as

$$
\beta=\frac{\mathrm{d}^{2} \boldsymbol{e}_{1}}{\mathrm{~d} s^{2}} \cdot \boldsymbol{e}_{3}
$$

It can be proved that the geodesic curvature of $\boldsymbol{I}_{1}$ is 0 , leading to the conclusion that the spherical indicatrix of $\boldsymbol{I}_{1}$ is part of big circle of the unit sphere. The above phenomenon and proof were not mentioned in previous literature.

The striction curve of the constraint ruled surface is

$$
\boldsymbol{M}=\boldsymbol{f}+u \boldsymbol{I}_{1}
$$

where

$$
u=-\frac{\dot{\boldsymbol{f}} \cdot \dot{\boldsymbol{I}}_{1}}{\dot{\boldsymbol{I}}_{1} \cdot \dot{\boldsymbol{I}}_{1}}
$$

Figure 14 shows the striction curve of the constraint ruled surface where the base curve $f$ is the same one as that in Figure 13.

It is interesting to note that the striction curve consists of two separate branches. This explains the singularities in the striction curve of the constraint ruled surface.

\section{Conclusions}

This paper presented two assembly configurations of the Schatz linkage using differential geometry. Focused on the assembly configurations, the paper revealed for the first time two cycle phases of the linkage each of which completes the whole cycle of the ruled surface of the indicatrix along the coupler link when the input angle experiences half of the $2 \pi$ range. The study demonstrated that when the indicatrix $\boldsymbol{e}_{1}$ along the coupler link completes the cycle twice, the coupler link as a rigid body has a full rotation with input angle in the $2 \pi$ range.

The phase study led to investigation of the ruled surface of the coupler link with Euclidean invariants of both indicatrix and geodesic curvatures. It was found that the shape of the striction curve of the coupler-link ruled surface was alike to two raindrops 
tangentially connected. It was further found that the striction curve had two singular points while the Schatz linkage was known to not have any singularities.

The constraint ruled surface was generated with the base curve from the reciprocal screw and Euclidean invariants of the ruled surface directrix and striction curves. The striction curve of the ruled surface was found to have two disconnected branches. The geodesic curvature of the spherical indicatrix of the constraint ruled surface was found to be zero, indicating that the spherical indicatrix was a part of big circle of the unit sphere. This work revealed that its striction curve had one singular point, which might provide a clue to the shaking movement that was normally caused by dynamic discontinuity. The angles formed by the two tangents at the singular points of the striction curve implied the extent to which the shaking movement occurred. The study paves a foundation for the design of the Schatz linkage.

\section{Acknowledgements}

The authors gratefully acknowledge the support provided by the Innovative China-UK (ICUK) award (grant number KCL02).

\section{Conflict of interest}

None declared.

\section{References}

1. Ku J, Mraz R, Baker N, et al. A data glove with tactile feedback for FMRI of virtual reality experiments. CyberPsychol Behav 2003; 6(5): 497-508.

2. Sarrus PT. Note Sur La Transformation Des Mouvements Rectilignes Alternatifs,En Mouvements Circulaires, Et Reciproquement. Académie Sci 1853; 36(1): 1036-1038.

3. Bennett GT. A new mechanism. Engineering 1903; 76(999999): 777-778.

4. Bennett GT. The parallel motion of Sarrus and some allied mechanisms. Philos Mag 1905; 6(9): 803-810.

5. Bennett GT. The skew isogram mechanism. Proc Lond Math Soc 1914; 23(1): 151-173.

6. Bricard R. Leçons De Cinématique. Tome II Cinématique Appliquée, 1927, pp.7-12.

7. Myard FE. Contribution A La Géométrie. Soc Mathémat de France 1931; 59(1): 183-210.

8. Goldberg M. New five-bar and six-bar linkages in three dimensions. ASME 1953; 65(1): 649-663.

9. Altmann PG. Communications to Grodzinski, P. And M'ewen, E, Link mechanisms in modern kinematics. Proc Instn Mech Engrs 1954; 168(37): 889-896.

10. Waldron KJ. Hybrid overconstrained linkages. $J$ Mech 1968; 3(2): 73-78.

11. Schatz P. Rhythmusforschung Und Technik. Stuttgart: Verlag Freies Geistesleben, 1975.
12. Mavroidis C and Roth B. Analysis of overconstrained mechanisms. ASME Trans J Mech Des 1995; 117: 69-74.

13. Baker JE. A curious new family of overconstrained sixbars. ASME Trans J Mech Des 2005; 127(4): 602-606.

14. Cui L and Dai JS. Axis constraint analysis and its resultant 6R double-centered overconstrained mechanisms. Trans ASME J Mech Robot 2011; 3(3): 031004.

15. Yu HC. Geometrical investigation of general octahedral linkages and the turbula. Mech Mach Theory 1980; 15(6): 463-478.

16. Baker JE, Duclong $\mathrm{T}$ and Khoo PSH. On attempting to reduce undesirable inertial characteristics of the Schatz mechanism. ASME Trans J Mech Des 1982; 104(2): 192-205.

17. Lee CC. Analysis and synthesis of schatz six-revolute mechanisms. JSME Int J 2000; 43(1): 80-91.

18. Lee C-C and Dai JS. Configuration analysis of the Schatz linkage. Proc IMechE, Part C: J Mechanical Engineering Science 2003; 217(7): 779-786.

19. Horne A. Mathmatical modelling and simulation of the Schatz linkage, MSc Dissertation, University of London, UK.

20. Dai JS. An historical review of the theoretical development of rigid body displacements from Rodrigues parameters to the finite twist. Mech Mach Theory 2006; 41(1): 41-52.

21. Dai JS, Huang Z and Lipkin H. Mobility of overconstrained parallel mechanisms. Journal of Mechanical Design, Trans. ASME 2006; 128(1): 220-229.

22. Sasaki S. Differential geometry, Tokyo: Kyoritsu Press.

23. Mccarthy JM and Roth B. The curvature theory of line trajectories in spatial kinematics. ASME Trans J Mech Des 1981; 103(4): 23-30.

24. Mccarthy JM. The instantaneous kinematics of line trajectories in terms of a kinematic mapping of spatial rigid motion. ASME Trans J Mech Transm Automat Des 1987; 109(1): 95-100.

25. Mccarthy JM. On the scalar and dual formulations of the curvature theory of line trajectories. ASME Trans $J$ Mech Transm Automat Des 1987; 109(1): 101-106.

26. Pottmann $\mathrm{H}$ and Wallner J. Computational line geometry, New York: Springer.

27. Cui L, Wang D and Dai JS. Kinematic geometry of circular surfaces with a fixed radius based on Euclidean invariants. ASME Trans J Mech Des 2009; 131(10): 101009 1-8.

28. Cui L and Dai JS. A Darboux frame based formulation of spin-rolling motion of rigid objects with point contact. IEEE Trans Robot 2010; 26(2): 383-388.

29. Lipschutz MM. Schaum's outline of differential geometry. Schaum's Outlines, 1969.

30. Dai JS and Jones JR. Null space construction using cofactors from a screw algebra context. Proc Roy Soc Lond Math Phys Eng Sci 2002; 458(2024): 1845-1866. 